Versicherten Wahlmöglichkeiten zwischen verschiedenen aufzahlungsfreien Hilfsmitteln einzuräumen. Für Hilfsmittel mit hohem individuellen Anpassungsbedarf darf es künftig keine Ausschreibung geben.

_Die Krankenkassen müssen die Einhaltung der gesetzlichen und vertraglichen Pflichten der Leistungserbringer mit Auffälligkeits- und Stichprobenprüfungen kontrollieren. Der GKV-Spitzenverband wird verpflichtet, bis zum 30. Juni 2017 Rahmenempfehlungen zur Vertragskontrolle abzugeben.

_Leistungserbringer müssen Versicherte künftig beraten, welche Hilfsmittel und zusätzlichen Leistungen innerhalb des Sachleistungssystems für sie geeignet sind und somit von den Krankenkassen als Regelleistung bezahlt werden.

_ Auch die Krankenkassen werden zu einer verbesserten Beratung der Versicherten über ihre Rechte bei der Hilfsmittelversorgung verpflichtet. Bei der Versorgung mit Hilfsmitteln, für die zuvor eine Genehmigung einzuholen ist, müssen die Krankenkassen über ihre Vertragspartner und die wesentlichen Inhalte der abgeschlossenen Verträge informieren. Die Krankenkassen müssen über die von ihnen abgeschlossene Verträge im Internet berichten. Damit können Versicherte die Angebote verschiedener Krankenkassen vergleichen.

_Die Ausnahmeregelung für einen Leistungsanspruch auf Brillengläser wird erweitert: Künftig erhalten auch die Versicherten, die wegen einer Kurz- oder Weitsichtigkeit Gläser mit einer Brechkraft von mindestens 6 Dioptrien oder wegen einer Hornhautverkrümmung von mindestens $4 \mathrm{Di}$ optrien benötigen, einen Anspruch auf Kostenübernahme in Höhe des vom GKV-Spitzenverband festgelegten Festbetrags beziehungsweise des von ihrer Krankenkasse vereinbarten Vertragspreises. Nach derzeitiger Rechtslage werden die Kosten für Brillengläser nur für Kinder und Jugendliche übernommen. Volljährige Versicherte haben nur dann einen Leistungsanspruch, wenn sie auf beiden Augen eine extreme Sehschwäche auf-

\title{
FRAGE
}

\section{Fahrtzeit in der ambulanten Pflege}

Zählt die Fahrtzeit der ambulanten Pflegekräfte eigentlich zur bezahlten Arbeitszeit?

Der Begriff Fahrtzeit wird - wie der Begriff der Wegezeit - im Arbeitsrecht nicht einheitlich verwendet. Deshalb ist bei der Frage zu unterscheiden, welche Strecke konkret mit der Fahrtzeit gemeint ist, bevor die Frage nach der Vergütung gestellt werden kann.

Die Zeit, die der Arbeitnehmer für den Weg von seiner Wohnung zum Betrieb benötigt, ist die Wegezeit. Die Wegezeit ist grundsätzlich nicht Teil der Arbeitsleistung und sie wird grundsätzlich nicht vergütet (BAG 21.12.2006, Neue Zeitschrift für Arbeitsrecht 2008, S. 136). Eine Ausnahme vom Grundsatz, dass die Wegezeit nicht vergütet wird, stellt die Rufbereitschaft dar: Bei der Rufbereitschaft zählt die Fahrt des Arbeitnehmers zum Betrieb zur Arbeitszeit (Arbeitsgericht Marburg 04.11.2003, 2 Ca 212/03). Fährt eine ambulante Pflegekraft also mit ihrem Fahrrad von ihrer Wohnung zum Büro des Pflegedienstes, von dem aus sie dann die Kunden anfährt, zählt die Fahrt von zu Hause zum Pflegedienstbüro nicht zur Arbeitszeit und wird nicht vergütet. Und wie steht es um die Fahrtzeiten vom Pflegebüro zu den pflegebedürftigen Kunden? Das BAG hat in einer Entscheidung vom 12.12.2012 klargestellt, dass Fahrtzeiten vom Betrieb zu einer auswärtigen Arbeitsstelle als Arbeitszeit zu bewerten ist (5 AZR 355/12). Das BAG ergänzte, dass mit der Qualifizierung der Fahrtzeit als Arbeitszeit noch nichts über die Höhe der Vergütung ausgesagt ist. Durch Arbeitsvertrag oder Tarifvertrag kann, so das BAG, geregelt werden, dass Fahrtzeiten vom Betrieb zur Einsatzstätte anders vergütet werden als die eigentliche Tätigkeit. Dabei ist jedoch das Mindestlohngesetz einzuhalten. In seinem Urteil vom 10.09.2015 (C-266/14) hat der Europäische Gerichtshof die Rechtslage für eine weitere Fallkonstellation geklärt: Wenn ein Arbeitnehmer keinen festen oder gewöhnlichen Arbeitsort hat, zählt bereits das Zurücklegen der Strecke von der Wohnung zum Einsatzort zur Arbeitszeit. Würde es für eine ambulante Pflegekraft also kein Büro des Pflegedienstes geben, das sie regelmäßig vor Beginn ihrer Pflegediensttour ansteuert, zählt die Fahrt von ihrer Wohnung zur Wohnung des ersten Kunden bereits zur vergütungspflichtigen Arbeitszeit. Dies wirkt sich auch auf die gesetzlich zulässige Arbeitszeit aus.

\section{Martina Weber}

Volljuristin (Ass. jur.)

weisen und ihre Sehleistung auf dem besseren Auge bei bestmöglicher Korrektur höchstens $30 \%$ erreicht.

-Die Krankenkassen werden verpflichtet, mit den Verbänden der Heilmittelerbringer Verträge über Modellvorhaben zur sogenannten Blankoverordnung abzuschließen. Dabei wird das Heilmittel weiterhin durch den Arzt verordnet, der Heilmittelerbringer bestimmt aber die Auswahl und die Dauer der Therapie sowie die Frequenz der Behandlungseinheiten. Zwei Modellvorhaben zur Blankoverordnung haben in den letzten Jahren bereits stattgefunden. Um zu entscheiden, ob diese Versorgungsform für eine Überführung in die Regelversorgung geeignet ist, sind aber mehr In- formationen notwendig. Deshalb soll es in jedem Bundesland ein Modellvorhaben geben.

_Das Präqualifizierungsverfahren, in dem Apotheken, Sanitätshäuser, orthopädietechnische Betriebe und andere Anbieter von Hilfsmitteln ihre grundsätzliche Eignung für Vertragsabschlüsse mit den Krankenkassen nachweisen können, wird weiterentwickelt. Die Deutsche Akkreditierungsstelle $\mathrm{GmbH}$ (DAkkS) begutachtet, akkreditiert und überwacht in $\mathrm{Zu}$ kunft die derzeit bundesweit rund 30 Präqualifizierungsstellen.

Das Gesetz bedarf nicht der Zustimmung des Bundesrates.

www.bundesgesundheitsministerium.de 